\title{
Multi-attribute Decision-making Model of Grey Target for Information System Evaluation
}

\author{
Sha Fu*, Guang Sun, Yezhi Xiao \\ Department of Information Management, Hunan University of Finance and Economics, China
}

Copyright $(2016$ by authors, all rights reserved. Authors agree that this article remains permanently open access under the terms of the Creative Commons Attribution License 4.0 International License

\begin{abstract}
A kind of multi-attribute grey-target decision model based on positive-negative target center is put forward aimed at the complexity and uncertainty of the actual decision environment. Firstly, the optimal effect vector and the worst effect vector of the grey-target decision are respectively defined as the positive and negative clouts; secondly, the spatial projection distance between the positive and negative clouts shall be comprehensively considered, and the off-target distance is the basis of the vector analysis for space and then a new comprehensive target-eyes distance shall be acquired; then, build the goal programming model for objective function in accordance with comprehensive target-eyes distance, and make solution by that to get the index weight. At last, verify the feasibility and validity of the proposed grey-target decision model by the optional instance analysis of information system.
\end{abstract}

Keywords Multi-attribute, Positive and Negative Clouts, Grey Target Decision, Comprehensive Target-eyes Distance, Index Weight, Goal Programming

\section{Introduction}

In recent years, the multi-attribute decision problem has become the hot issue investigated and discussed by experts and scholars at home and abroad and rich academic research results have been formulated. The grey system theory has been used to solve technology evaluation, project option, and supply chain management relative decision problems with grey-target decision as an important component of grey decision method. Integrating research literature at home and abroad, we can see that many experts and scholars have conducted theoretical researches for the classical grey-target decision method from different aspects and got some research results. Such as: William Ho et al.[1] proposed the literature of the multi-criteria decision making approaches for supplier evaluation and selection. This study not only provides evidence that the multi-criteria decision making approaches are better than the traditional cost-based approach, but also aids the researchers and decision makers in applying the approaches effectively. Eshlaghy and Razi [2] presented an integrated framework for project selection and project management approach using grey-based k-means and genetic algorithms. The proposed approach of this study first cluster different projects based on k-means algorithm and then ranks R\&D projects by grey relational analysis model. Luo and Wang [3], Based on the grey system theory and methods, the grey-target decision-making problem is discussed, in which the attribute values are grey numbers and the maximum probability of the value of grey number is known. Wann-Yih $\mathrm{Wu}$ et al. [4] presented an alternative evaluation procedure to help retailers, especially hyper marketers, make a location decision by using the grey multi-objective decision method. Chaang-Yung and Kun-Li [5] used six financial indicators to classify twenty items of financial ratios as research variables through the Globalization Grey Relational Analysis, to find the significant financial ratio variables and other financial indicators affecting the financial performance of venture capital enterprises in Taiwan, and it applied Grey Decision-Making to arrange the total performances of the sample venture capital enterprises in order. Liu et al.[6] proposed a novel multi-attribute grey target decision model and demonstrated with a practical case study. Dai and Li [7] introduced conceptions about group positive and negative clouts and group deviation degree of target center and put forward group decision methods about deviation degree of target center with grey multi-attribute for a kind of group decision problem that the attribute value, attribute weight and decider's weight all are interval grey numbers. Chen and Xie [8] studied the traditional incompatibility problem of polarity reversal of grey target and proved the existence and occurring probability of incompatibility by formulating a special index sequence of moderation value. Wang et al.[9] considered the impact of relevance, different dimensions, and importance differences of all indexes on the decision effect, and used the weighted mahalanobis distance to improve the traditional grey-target decision method, which avoided the impact of relevance, different dimensions, and importance differences of all indexes and the incompatibility 
of grey-target change. The above researches provide some ideas for the solution of the grey-target decision problem, but we can also find that researches for the grey-target decision problem with the decision form in the form of interval grey numbers and the undetermined index weight are relatively less, so corresponding grey-target decision models are put forward in the paper to meet the requirements of such decision.

\section{Problem Description}

Set $n$ decision schemes for multi-attribute decision problem and formulate decision scheme set $A=\left\{A_{1}, A_{2}, \cdots, A_{n}\right\} . m$ evaluation indexes (attributes) are formed the attribute set $C=\left\{C_{1}, C_{2}, \cdots, C_{m}\right\}$. The scheme $A_{i}$ 's attribute value for index $C_{j}$ is $x_{i j}(\otimes) \in\left[x_{i j}, \bar{x}_{i j}\right]$, of which, $0 \leq x_{i j} \leq \bar{x}_{i j}$; if $i=1,2, \cdots, n ; j=1,2, \cdots, m$, the scheme set $A$ 's effect measure matrix $X$ for attribute set $C$ is:

\begin{tabular}{ccccc}
\hline & $C_{1}$ & $C_{2}$ & $\cdots$ & $C_{m}$ \\
\hline$A_{1}$ & $x_{11}(\otimes)$ & $x_{12}(\otimes)$ & $\cdots$ & $x_{1 m}(\otimes)$ \\
$A_{2}$ & $x_{21}(\otimes)$ & $x_{22}(\otimes)$ & $\cdots$ & $x_{2 m}(\otimes)$ \\
$\vdots$ & $\vdots$ & $\vdots$ & $\ddots$ & $\vdots$ \\
$A_{n}$ & $x_{n 1}(\otimes)$ & $x_{n 2}(\otimes)$ & $\cdots$ & $x_{n m}(\otimes)$ \\
\hline
\end{tabular}

\section{Preliminaries}

\subsection{Grey-target Decision}

In 1982, China scholar Deng Ju-long professor has established the grey system theory. After that, the grey system theory has been widely applied and a series of grey decision methods have been produced, such as: grey-target decision. Grey-target decision is the non-uniqueness principle application and reflection of grey system theory on decision theory and its basic thought is: find the standard index sequence most proximal to the target value among a group of index sequences made up of decision object and call it the target center of the grey-target. Index sequences formed by other decision objects and target centers jointly constitute the grey-target and their grey correlation degree to target centers is referred to as target center proximity [10]. Evaluate and arrange the decision object mainly based on the magnitude of target center proximity.

\subsection{Distance and Possibility Degree Formula of Interval Grey Number}

In grey system theory, the number with it's probably range known and its explicit value unknown is referred to as the grey number. The grey number is the basic unit of grey system. The grey number with the lower bound $\underline{a}$ and the upper bound $\bar{a}$ is the interval grey number, which is denoted by $a(\otimes) \in[a, \bar{a}]$.

Definition 1: If there are two interval grey numbers $a(\otimes) \in[\underline{a}, \bar{a}]$ and $b(\otimes) \in[\underline{b}, \bar{b}], k$ is the arithmetic number, and the calculation principle is as follows [11]:
1) $\quad a(\otimes)+b(\otimes) \in[\underline{a}+\underline{b}, \bar{a}+\bar{b}]$;
2) $a(\otimes) b(\otimes) \in[\min \{\underline{a} \underline{b}, \underline{a} \bar{b}, \bar{a} \underline{b}, \bar{a} \bar{b}\}$, $\max \{a \underline{b}, \underline{a} \bar{b}, \bar{a} \underline{b}, \bar{a} \bar{b}\}]$
3) $k+a(\otimes) \in[k+\underline{a}, k+\bar{a}]$;
4) $k a(\otimes) \in[k \underline{a}, k \bar{a}]$.

Definition 2: If there are two interval grey numbers $a(\otimes) \in[\underline{a}, \bar{a}]$ and $b(\otimes) \in[\underline{b}, \bar{b}]$, the distance of the interval grey number $a(\otimes)$ and $b(\otimes)$ is [12]:

$$
L(a(\otimes), b(\otimes))=2^{-\frac{1}{2}}\left[(\underline{a}-\underline{b})^{2}+(\bar{a}-\bar{b})^{2}\right]^{\frac{1}{2}}
$$

Definition 3: For the interval grey number $a(\otimes) \in[\underline{a}, \bar{a}]$ and $b(\otimes) \in[\underline{b}, \bar{b}]$, noted as $l_{a}=\bar{a}-\underline{a}, l_{b}=\bar{b}-\underline{b}$, then the

$$
p(a(\otimes) \geq b(\otimes))=\frac{\min \left\{l_{a}+l_{b}, \max (\bar{a}-\bar{b}, 0)\right\}}{l_{a}+l_{b}}
$$

is the possibility degree of $a(\otimes) \geq b(\otimes)$.

\section{Decision Model for Multi-attribute Grey-target}

\subsection{Decision Model Establishment for Multi-attribute Grey-target}

\subsubsection{Normalization Handling of Decision Matrix}

Since all attribute values of decision matrix have different measurement standards and measurement units, the following formula shall be used in the normalization handling for the uniform handling and the obtainment of the normalization decision matrix. Common attribute types of the multi-attribute decision problem are benefit and cost type. For the benefit attribute type, the larger the value is, the better the value is; while the cost attribute is on the contrary. If the $I_{1}$ and $I_{2}$ respectively is benefit type and cost type attribute.

For the benefit type attribute, there is: 


$$
\underline{z}_{i j}=\frac{\underline{x}_{i j}}{\sqrt{\sum_{i=1}^{n}\left(\bar{x}_{i j}\right)^{2}}}, \bar{z}_{i j}=\frac{\bar{x}_{i j}}{\sqrt{\sum_{i=1}^{n}\left(\underline{x}_{i j}\right)^{2}}}
$$

Of which, $i=1,2, \cdots, n ; j \in I_{1}$

For cost type attribute, there is:

$$
\underline{z}_{i j}=\frac{\left(1 / \bar{x}_{i j}\right)}{\sqrt{\sum_{i=1}^{n}\left(1 / \underline{x}_{i j}\right)^{2}}}, \bar{z}_{i j}=\frac{\left(1 / \underline{x}_{i j}\right)}{\sqrt{\sum_{i=1}^{n}\left(1 / \bar{x}_{i j}\right)^{2}}}
$$

Of which, $i=1,2, \cdots, n ; j \in I_{2}$

\subsubsection{Grey-target Decision for Positive-negative Target Center}

Definition 4: If $z_{j}^{+}=\max \left\{\left(\underline{z}_{i j}+\bar{z}_{i j}\right) / 2 \mid 1 \leq i \leq n\right\}$, $(j=1,2, \cdots, m)$, its corresponding decision value is noted as $\left[\underline{z}_{i j}^{+}, \bar{z}_{i j}^{+}\right]$, which is referred to as

$$
z^{+}=\left\{z_{1}^{+}, z_{2}^{+}, \cdots, z_{m}^{+}\right\}=\left\{\left[\underline{z}_{i 1}^{+}, \bar{z}_{i 1}^{+}\right],\left[\underline{z}_{i 2}^{+}, \bar{z}_{i 2}^{+}\right], \cdots,\left[\underline{z}_{i m}^{+}, \bar{z}_{i m}^{+}\right]\right\}
$$

the optimal effect vector of grey-target decision and is called positive target center.

Definition 5: If $z_{j}^{-}=\min \left\{\left(\underline{z}_{i j}+\bar{z}_{i j}\right) / 2 \mid 1 \leq i \leq n\right\}$, $(j=1,2, \cdots, m)$, its corresponding decision value is $\left[\underline{z}_{i j}^{-}, \bar{z}_{i j}^{-}\right]$, which is referred to as

$$
z^{-}=\left\{z_{1}^{-}, z_{2}^{-}, \cdots, z_{m}^{-}\right\}=\left\{\left[\underline{z}_{i 1}^{-}, \bar{z}_{i 1}^{-}\right],\left[\underline{z}_{i 2}^{-}, \bar{z}_{i 2}^{-}\right], \cdots,\left[\underline{z}_{i m}^{-}, \bar{z}_{i m}^{-}\right]\right\}
$$

the worst effect vector of the grey-target decision and is called the negative target center.

Where, index weight is $w=\left(w_{1}, w_{2}, \cdots, w_{m}\right)$ and

$$
\sum_{j=1}^{m} w_{j}=1 \text {. }
$$

Definition 6: Call

$$
\begin{aligned}
& \varepsilon_{i}^{+}=2^{-\frac{1}{2}}\left[w_{1}\left(\underline{z}_{i 1}-\underline{z}_{i 1}^{+}\right)^{2}\right]+ \\
& \left.+w_{1}\left(\bar{z}_{i 1}-\bar{z}_{i 1}^{+}\right)^{2}+\cdots+w_{m}\left(\bar{z}_{i m}-\bar{z}_{i m}^{+}\right)^{2}\right]^{\frac{1}{2}}
\end{aligned}
$$

as the positive off-target distance of effect vector $z_{i}$. Call

$$
\begin{aligned}
& \varepsilon_{i}^{-}=2^{-\frac{1}{2}}\left[w_{1}\left(\underline{z}_{i 1}-\underline{z}_{i 1}^{-}\right)^{2}\right]+ \\
& \left.+w_{1}\left(\bar{z}_{i 1}-\bar{z}_{i 1}^{-}\right)^{2}+\cdots+w_{m}\left(\bar{z}_{i m}-\bar{z}_{i m}^{-}\right)^{2}\right]^{\frac{1}{2}}
\end{aligned}
$$

as the negative off-target distance of effect vector $z_{i}$.

\section{Definition 7: Call}

$$
\begin{aligned}
& \varepsilon_{i}^{0}=2^{-\frac{1}{2}}\left[w_{1}\left(\underline{z}_{i 1}^{+}-\underline{z}_{i 1}^{-}\right)^{2}\right]+ \\
& \left.+w_{1}\left(\bar{z}_{i 1}^{+}-\bar{z}_{i 1}^{-}\right)^{2}+\cdots+w_{m}\left(\bar{z}_{i m}^{+}-\bar{z}_{i m}^{-}\right)^{2}\right]^{\frac{1}{2}}
\end{aligned}
$$

as the positive-negative target center interval.

Form definitions in the literature [13], the distance $\varepsilon_{i}^{+}, \varepsilon_{i}^{-}$ and $\varepsilon_{i}^{0}$ fall upon the same straight line or enclose a triangle. Therefore, the projection size of the positive off-target distance the connection line between positive and negative clouts shall be used to acquire the optimal solution for the event. So, the larger the projection is, the better the solution is. If the included angle of the connection line between the positive and negative clouts is $\theta$, we can know that in accordance with cosine law

$$
\left(\varepsilon_{i}^{+}\right)^{2}+\left(\varepsilon_{i}^{0}\right)^{2}-2 \varepsilon_{i}^{+} \varepsilon_{i}^{0} \cos \theta=\left(\varepsilon_{i}^{-}\right)^{2}
$$

Because the positive off-target distance $\varepsilon_{i}^{+}$and negative off-target distance $\varepsilon_{i}^{-}$all are vectors, and if the projection of off-target distance on the connection line between positive and negative clouts is taken into consideration, the comprehensive target-eyes distance $\varepsilon_{i}$ is:

$$
\varepsilon_{i}=\varepsilon_{i}^{+} \cos \theta=\frac{\left(\varepsilon_{i}^{+}\right)^{2}+\left(\varepsilon_{i}^{0}\right)^{2}-\left(\varepsilon_{i}^{-}\right)^{2}}{2 \varepsilon_{i}^{0}}
$$

The positive and negative clouts have been included in comprehensive target-eyes distance calculation and the off-target distance is used as the vector to make the decision information more scientific and reasonable.

\subsubsection{Determination of Index Weight}

The entropy concept was firstly proposed by Mr. Rudolf Clausius, a German physicist, in 1865 and was then introduced into the information field by the father of information theory C.E. Shannon in 1948 via utilizing the "Information entropy" as a measure of the disorder degree of information. If the index weight sequence $w=\left(w_{1}, w_{2}, \cdots, w_{m}\right)$ is unknown, then the sequence is the grey connotation sequence and the grey entropy is defined as:

$$
H_{\otimes}(w)=-\sum_{j=1}^{m} w_{j} \ln w_{j}
$$

The $w_{j}(j=1,2, \cdots, m)$ shall be regulated in accordance with maximum entropy theory to reduce the uncertainty of the sequence $w=\left(w_{1}, w_{2}, \cdots, w_{m}\right)$ which means the maximization of $H_{\otimes}(w)$. At the same time, weight $w_{j}(j=1,2, \cdots, m) \quad$ regulation makes the overall comprehensive target-eyes distance minimum, so the following multi-objective optimization model can be established for this: 


$$
\left\{\begin{array}{l}
\min \sum_{i=1}^{n} \varepsilon_{i}=\sum_{i=1}^{n} \frac{\left(\varepsilon_{i}^{+}\right)^{2}+\left(\varepsilon_{i}^{0}\right)^{2}-\left(\varepsilon_{i}^{-}\right)^{2}}{2 \varepsilon_{i}^{0}} \\
\max H_{\otimes}(w)=-\sum_{j=1}^{m} w_{j} \ln w_{j} \\
\text { s.t. } \sum_{j=1}^{m} w_{j}=1, w_{j} \geq 0, j=1,2, \cdots, m
\end{array}\right.
$$

In order to solve such multi-objective optimization model, the above stated multi-objective optimization model shall be transformed into the single-objective optimization model according to the fair competitiveness of all schemes.

$$
\left\{\begin{array}{l}
\min \left\{\mu \sum_{i=1}^{n} \frac{\left(\varepsilon_{i}^{+}\right)^{2}+\left(\varepsilon_{i}^{0}\right)^{2}-\left(\varepsilon_{i}^{-}\right)^{2}}{2 \varepsilon_{i}^{0}}+(1-\mu) \sum_{j=1}^{m} w_{j} \ln w_{j}\right\} \\
\text { s.t. } \sum_{j=1}^{m} w_{j}=1, w_{j} \geq 0, j=1,2, \cdots, m
\end{array}\right.
$$

Where, $0<\mu<1$. Based on the fair competitiveness of objective function optimization, generally the $\mu=0.5$ [12] is taken. Solve the model by the Visual $\mathrm{C}++$ programming and obtain the index weight sequence $w=\left(w_{1}, w_{2}, \cdots, w_{m}\right)$. At last, substitute that into formula (10) and get comprehensive target-eyes distance $\varepsilon_{i}$. Arrange the alternative scheme in accordance with the $\varepsilon_{i}$ value. The smaller $\varepsilon_{i}$ is, the better the corresponding scheme is.

\subsection{Procedures of Multi- attribute Grey-target Decision}

In conclusion, the specific procedures of multi-attribute grey-target decision based on the positive and negative clouts are as follows:

Step 1 Use the formula (3) and (4) to conduct normalization handling for the decision matrix $X$ and obtain the normalization decision matrix $Z$;

Step 2 Use the formula (5) and (6) to respectively determine the positive and negative clouts of grey-target decision;

Step 3 Use the formula (7) and (8) to respectively determine the positive and negative off-target distance of effect vector $z_{i}$, and obtain the positive and negative clouts interval by calculation in accordance with the Formula (9).

Step 4 Solve the model by the single-objective optimization model shown in the Formula (13) and the software programming way and obtain the weight sequence $w=\left(w_{1}, w_{2}, \cdots, w_{m}\right) ;$

Step 5 Use the Formula (10) to determine the comprehensive target-eyes distance $\varepsilon_{i}$, and arrange all alternative schemes in accordance with the $\varepsilon_{i}$ value.

\section{Application Examples}

Demonstrate the applications of the above multi-attribute decision methods with decision attribute value of the interval grey number and uncertain attribute weight through examples. Take the integration service of E-government affair system purchase of an organization as an example, the purchaser shall select the best enterprise from the five domestic integrators of information system $\left(A_{1}, A_{2}, A_{3}, A_{4}, A_{5}\right)$ to complete the hardware and software construction and follow service of the E-government affair system. There are five main evaluation indexes (attributes) in accordance with the business requirements of the purchaser and the performance requirements of the E-government affair system: system quotation $\left(C_{1}\right)$, the committed time of the integration service completion $\left(C_{2}\right)$, usability, safety and expansibility performances of software system $\left(C_{3}\right)$, the research capacity and after-sale service capacity of enterprise $\left(C_{4}\right)$, and enterprise industry reputation $\left(C_{5}\right)$ [14]. Evaluation expert will respectively evaluate the five enterprises in all sides and the acquired data is shown in Table 1 after cleansing.

Table 1. Decision Matrix X of Interval Number

\begin{tabular}{lccccc}
\hline & $C_{1}$ & $C_{2}$ & $C_{3}$ & $C_{4}$ & $C_{5}$ \\
\hline$A_{1}$ & {$[8.20,8.64]$} & {$[1.8,2.1]$} & {$[8.2,8.7]$} & {$[2050,2240]$} & {$[0.85,0.90]$} \\
$A_{2}$ & {$[8.08,8.43]$} & {$[2.5,2.8]$} & {$[8.5,9.0]$} & {$[2140,2200]$} & {$[0.80,0.95]$} \\
$A_{3}$ & {$[7.66,7.89]$} & {$[2.0,2.5]$} & {$[8.0,8.5]$} & {$[1810,1900]$} & {$[0.75,0.85]$} \\
$A_{4}$ & {$[7.24,7.54]$} & {$[1.9,2.2]$} & {$[7.0,7.5]$} & {$[1760,1850]$} & {$[0.65,0.80]$} \\
$A_{5}$ & {$[8.85,9.26]$} & {$[2.7,3.1]$} & {$[8.0,9.0]$} & {$[1950,2040]$} & {$[0.80,0.90]$} \\
\hline
\end{tabular}

Step 1 among the above attributes, the $C_{1}, C_{2}$ and $C_{3}$ belong to the benefit attribute. Use the formula (3) to conduct normalization handling for decision matrix $X ; C_{4}$ and $C_{5}$ belong to the cost type attribute and the Formula (4) shall be used to conduct normalization handling to obtain the normalization decision matrix $Z$ which is as shown in the Table 2.

Table 2. Normalization Decision Matrix Z

\begin{tabular}{cccccc}
\hline & $C_{1}$ & $C_{2}$ & $C_{3}$ & $C_{4}$ & $C_{5}$ \\
\hline$A_{1}$ & {$[0.438,0.482]$} & {$[0.314,0.425]$} & {$[0.429,0.489]$} & {$[0.385,0.442]$} & {$[0.378,0.461]$} \\
$A_{2}$ & {$[0.432,0.470]$} & {$[0.436,0.567]$} & {$[0.444,0.506]$} & {$[0.392,0.424]$} & {$[0.358,0.489]$} \\
$A_{3}$ & {$[0.409,0.440]$} & {$[0.348,0.506]$} & {$[0.418,0.478]$} & {$[0.453,0.501]$} & {$[0.400,0.522]$} \\
$A_{4}$ & {$[0.387,0.420]$} & {$[0.331,0.445]$} & {$[0.366,0.422]$} & {$[0.466,0.515]$} & {$[0.425,0.602]$} \\
$A_{5}$ & {$[0.473,0.516]$} & {$[0.470,0.628]$} & {$[0.418,0.506]$} & {$[0.422,0.465]$} & {$[0.378,0.489]$} \\
\hline
\end{tabular}


Step 2 Use the Formulas (5) and (6) to respectively calculate the positive and negative clouts of grey-target decision.

$$
\begin{aligned}
z^{+} & =\{[0.473,0.516],[0.470,0.628],[0.444,0.506],[0.466,0.515],[0.425,0.602]\} \\
z^{-} & =\{[0.387,0.420],[0.314,0.425],[0.366,0.422],[0.385,0.424],[0.358,0.461]\}
\end{aligned}
$$

Step 3 Use the Formulas (7) and (8) respectively determines the positive and negative off-target distance of effect vector $z_{i}$.

Its positive off-target distance is:

$$
\left\{\begin{array}{l}
\varepsilon_{1}^{+}=2^{-\frac{1}{2}}\left[0.0024 w_{1}+0.0656 w_{2}+0.0005 w_{3}+0.0119 w_{4}+0.0223 w_{5}\right]^{\frac{1}{2}} \\
\varepsilon_{2}^{+}=2^{-\frac{1}{2}}\left[0.0038 w_{1}+0.0049 w_{2}+0.0139 w_{4}+0.0173 w_{5}\right]^{\frac{1}{2}} \\
\varepsilon_{3}^{+}=2^{-\frac{1}{2}}\left[0.0099 w_{1}+0.0296 w_{2}+0.0015 w_{3}+0.0004 w_{4}+0.0071 w_{5}\right]^{\frac{1}{2}} \\
\varepsilon_{4}^{+}=2^{-\frac{1}{2}}\left[0.0166 w_{1}+0.0526 w_{2}+0.0133 w_{3}\right]^{\frac{1}{2}} \\
\varepsilon_{5}^{+}=2^{-\frac{1}{2}}\left[0.0007 w_{3}+0.0044 w_{4}+0.015 w_{5}\right]^{\frac{1}{2}}
\end{array}\right.
$$

Its negative off-target distance is:

$$
\left\{\begin{array}{l}
\varepsilon_{1}^{-}=2^{-\frac{1}{2}}\left[0.0064 w_{1}+0.0085 w_{3}+0.0003 w_{4}+0.0004 w_{5}\right]^{\frac{1}{2}} \\
\varepsilon_{2}^{-}=2^{-\frac{1}{2}}\left[0.0045 w_{1}+0.035 w_{2}+0.0133 w_{3}+0.0008 w_{5}\right]^{\frac{1}{2}} \\
\varepsilon_{3}^{-}=2^{-\frac{1}{2}}\left[0.0009 w_{1}+0.0078 w_{2}+0.0059 w_{3}+0.0107 w_{4}+0.0055 w_{5}\right]^{\frac{1}{2}} \\
\varepsilon_{4}^{-}=2^{-\frac{1}{2}}\left[0.0007 w_{2}+0.0149 w_{4}+0.0246 w_{5}\right]^{\frac{1}{2}} \\
\varepsilon_{5}^{-}=2^{-\frac{1}{2}}\left[0.0166 w_{1}+0.0656 w_{2}+0.0098 w_{3}+0.0031 w_{4}+0.0012 w_{5}\right]^{\frac{1}{2}}
\end{array}\right.
$$

Interval of positive and negative clouts is:

$$
\begin{aligned}
& \varepsilon^{0}=2^{-\frac{1}{2}}\left[0.0166 w_{1}+0.0656 w_{2}+\right. \\
& \left.+0.0133 w_{3}+0.0149 w_{4}+0.0246 w_{5}\right]^{\frac{1}{2}}
\end{aligned}
$$

Step 4 Solve the model by using the single-objective optimization model determined by the Formula (13) and apply the software programming and obtain the index weight by calculation.

$$
\begin{gathered}
w_{1}=0.212, w_{2}=0.150, w_{3}=0.229, \\
w_{4}=0.216, w_{5}=0.193
\end{gathered}
$$

Step 5 Use the Formula (10) to determine the comprehensive target-eyes distance $\varepsilon_{i}$ and arrange all alternative schemes in accordance with the $\varepsilon_{i}$ value.

$$
\begin{gathered}
\varepsilon_{1}=0.0867, \varepsilon_{2}=0.0517, \varepsilon_{3}=0.0603, \\
\varepsilon_{4}=0.0697, \varepsilon_{5}=0.0269
\end{gathered}
$$

Thus $\varepsilon_{5}<\varepsilon_{2}<\varepsilon_{3}<\varepsilon_{4}<\varepsilon_{1}$ can be obtained, so, the arrangement result is: $\varepsilon_{5} \succ \varepsilon_{2} \succ \varepsilon_{3} \succ \varepsilon_{4} \succ \varepsilon_{1}$. Such result is basically consistent with the conclusion in literature [14] by calculation and analysis and such method is proved to be feasible and effective. We can see from its specific procedures and process that the method put forward in the paper is easy to be actually operated and can better meet the practical application requirements in comparison with methods used in the references.

\section{Conclusions}

Grey-target decision is one of the important methods to solve the multi-attribute decision problems. The paper formulates the decision model of multi-attribute grey-target based on the positive and negative clouts, and introduces the conceptions about positive-negative target center and positive and negative off-target distance. Put forward the calculation method of comprehensive target-eyes distance on that basis and by combining the spatial analysis and use the size of the comprehensive target-eyes distance to arrange advantages and disadvantages of all schemes. Provide a kind of scientific and practical decision methods for the solution of the grey-target decision problem with decision information of interval grey number and verify feasibility and validity of constructed model by the instance analysis.

\section{Acknowledgements}

This study was supported by the Scientific Research Fund of Hunan Provincial Education Department (No. 14C0184), by the Hunan Province Philosophy and Social Science 
Foundation (No. 14YBA065), supported by the construct program of the key discipline in Hunan province.

\section{REFERENCES}

[1] William Ho, Xiaowei Xu, Prasanta K. Dey, "Multi-criteria decision making approaches for supplier evaluation and selection: A literature review", European Journal of Operational Research, vol. 202, no. 1, pp. 16-24, April 2010.

[2] Abbas Toloie Eshlaghy, Farshad Faezy Razi, "A hybrid grey-based k-means and genetic algorithm for project selection", International Journal of Business Information Systems, vol. 18, no. 2, pp. 141-159, January 2015.

[3] Dang Luo, Xia Wang, “The multi-attribute grey target decision method for attribute value within three-parameter interval grey number", Applied Mathematical Modelling, vol.36, no. 5, pp. 1957-1963, May 2012.

[4] Wann-Yih Wu, Chuen-Jing Bai, Omprakash K. Gupta. “A hypermarket site selection model using the grey multi-objective decision method", International Journal of Logistics Systems and Management, vol. 2, no. 1, pp. 68-77, 2006.

[5] Chaang-Yung Kung, Kun-Li Wen, "Applying Grey Relational Analysis and Grey Decision-Making to evaluate the relationship between company attributes and its financial performance-A case study of venture capital enterprises in Taiwan", Decision Support Systems, vol.43, no.3, pp. 842-852, April 2007.

[6] Sifeng Liu, Bin Xu, Jeffrey Forrest, Ye Chen, "On Uniform Effect Measure Functions and a Weighted Multi-attribute
Grey Target Decision Model", The Journal of Grey System, vol. 25 ,no. 1, pp. 1-11, 2013.

[7] Dai Wen-zhan, Li Jiu-liang. "Off-target deviation degree method for grey multi-attribute group decision-making", Systems Engineering Theory \& Practice, vol. 34, no. 3, pp. 787-792, March 2014.

[8] Chen Yong-ming, Xie Hai-ying. "Test of the inconsistency problem on Deng's grey transformation by simulation", Systems Engineering and Electronics, vol. 29, no. 8, pp. 1285-1287, August 2007.

[9] Wang Z X, Dang Y G, Yang H. "Improvements on decision method of grey target", Systems Engineering and Electronics, vol. 31, no. 11, pp. 2634-2636,November 2009.

[10] Deng J L . Grey Prediction an grey decision. Wuhan: Press of Huazhong University of Science \& Technology, 2002: 56-61

[11] B Zeng, SF Liu, C Li, JM Chen, "Grey target decision making model of interval grey number based on cobweb area", Systems Engineering and Electronics, vol.35, no.11, pp. 2329-2334, November 2013.

[12] SongJie, Dang Yao-guo, Wang Zheng-xin, ZhangKe. "New decision model of grey target with both the positive clout and the negative clout", Systems Engineering Theory \& Practice, vol. 30, no. 10, pp. 1822-1827, October 2010.

[13] Luo Dang. "Multi-objective grey target decision model based on positive and negative clouts", Control and Decision, vol. 28, no. 2, pp. 241-246, February 2013.

[14] Fu Sha, Zhou Hang-jun, Liu Chao-qun, "Interval number multi-attribute decision making VIKOR extension method", Computer Engineering and Applications, vol. 49, no. 20, pp. 206-209, October 2013. 